\title{
Vertebrobasilar Dolichoectasia in a patient with recurrent Sudden Sensorineural Hearing Loss. Diagnostic approach and dilemmas
}

\author{
Eleni Zoe Gkoritsa ${ }^{1 *}$ and Athanasios A Plotas ${ }^{2}$ \\ ${ }^{1}$ Otorhinolaryngology Private Practice, Greece \\ ${ }^{2}$ CT \& MRI PELOPONNESOS Diagnostic Imaging Centre, Greece \\ *Corresponding author: Eleni Zoe Gkoritsa, University of Athens, 21, Petrou Mpoua str. Tripoli, Greece. \\ To Cite This Article: Eleni Zoe Gkoritsa, Athanasios A Plotas, Vertebrobasilar Dolichoectasia in a patient with recurrent Sudden Sensorineural \\ Hearing Loss. Diagnostic approach and dilemmas. Am J Biomed Sci \& Res. 2021 - 12(1). AJBSR.MS.ID.001715.
}

DOI: 10.34297/AJBSR.2021.12.001715

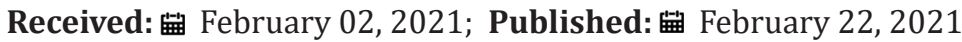

\begin{abstract}
Case Description: A fifty-five-year-old man had a recurrence of Sudden Sensorineural Hearing Loss (SSNHL) five months after the first incident which had left a residual moderate high frequency hearing loss and mild tinnitus. The recurrence involved mainly the low frequencies. He had a full audiovestibular test battery and MRI. On MRI he was diagnosed with Vertebrobasilar Dolichoectasia (VBD). Audiovestibular examination showed a cochlear hearing loss. He was treated with per os methylprednisolone. Low frequencies recovered almost fully.

Discussion: Vertebrobasilar Dolichoectasia i.e. the elongation, dilatation and tortuosity of the vertebrobasilar segment can have possible impacts on the audiovestibular system either by direct nerve compression of by blood flow disturbances. A thorough audiometric and imaging investigation was done, in order to determine to what extent VBD could be the cause of a recurrent hearing loss.

Conclusion: In the present case VBD looks unlikely to have caused the hearing loss. However due to being a potentially hazardous clinical entity clinical awareness is mandatory. In view of the uncommon audiological configuration and recurrence, further investigations are recommended.

Keywords: Basilar artery, Vertebrobasilar Dolichoectasia, Sudden Sensorineural Hearing Loss

Abbreviations: BA: Basilar artery; VA: Vertebral artery; AICA: Anterior Inferior Cerebellar Artery; VBD: Vertebrobasilar Dolichoectasia; SSNHL: Sudden Sensorineural Hearing Loss; MRI: Magnetic Resonance Imaging; CT: Computed Tomography; DSA: Digital Subtraction Angiography; PTA: Playtone Audiometry; ABR: Auditory Brainstem Responses; ECochG: Electrocochleography, p.o. per os.
\end{abstract}

\section{Introduction}

The basilar artery (BA) is the main source of posterior cerebral blood circulation. Consequently pathological entities leading to disturbance of blood flow in a vital part of the brain could be detrimental. The anatomy and normal measures of the vertebrobasilar system have been studied extensively in the past decades [1]. The most valid criteria for abnormality concerning the dimensions and location of the vertebrobasilar system, are Smoker's criteria [2] which are based on Computed tomography
(CT) and Digital Subtraction Angiography (DSA) measurements, but they are practically the same for MRI [3].

Vertebrobasilar dolichoectasia (VBD) is a vascular abnormality characterized by elongation, dilatation and/or tortuosity of the vertebrobasilar segment. Estimates of the prevalence of VBD range from $0.2 \%$ to $4.4 \%$ but sound population data are lacking [4]. Clinical expression of VBD can vary from asymptomatic (VBD detected incidentally) to stroke (hemorrhagic or ischemic) which 
is the most common manifestation of VBD. A 11 year follow up study [5] reported an incidence of $48 \%$ for stroke followed by compressive symptoms of cranial nerves at an incidence of $20 \%$ and hydrocephalus at an incidence of $1,2 \%$. Cranial nerves can be compressed either as an effect of immediate contact with the artery or as a mass effect. The most frequently involved cranial nerve roots are the facial (VII) and trigeminal (V)triggering hemifacial spasm or trigeminal neuralgia [2]. Cases of oculomotor (III) or abducens (VI) compression have been reported as well. Regarding audio vestibular nerve (VIII) compression by VBD, reports are very few. Moreover, Maruyama et al. [6] reviewing MRI findings in patients with audio vestibular symptoms who were diagnosed with VBD, introduced the question of possible existence of confounding factors leading to occurrence of Idiopathic Sudden Sensorineural Hearing Loss (ISSNHL) and/ or Canal Paresis in patients with VBD. The present case report is aiming to present a case of a 55-year-old man with recurrent hearing loss in the left ear who was diagnosed with VBD on MRI, to exhibit the possibilities of new diagnostic MRI modalities and how to combine audiovestibular test battery and imaging in order to answer a diagnostic dilemma.

\section{Case Report}

A 55-year-old man consulted the Otorhinolaryngology practice reporting sudden hearing loss with tinnitus in the left ear. This was the second incident, a previous one having occurred five months earlier. He was then treated by another physician. Playtone Audiometry (PTA) both times showed a hearing loss of both the low and the high frequencies (Figure 1A) preserving the mid frequencies at normal threshold. The first episode was treated by per os methylprednisolone at tapered doses. The low frequencies were restored back to normal, whereas the high frequencies improved slightly. Figure 1A shows the first and last (forty days later) audiograms of the first hearing loss incident. No imaging was done at the time of the first SSNHL episode. Four years before the first episode the patient had had an episode of Benign Paroxysmal Positional Vertigo. Playtone Audiometry at that time showed normal hearing thresholds in both ears. The patient has a history of hypertension and hyperlipidemia for which he has been on medication for the last four years.

The patient was first seen in our office when the second SSHNL incident occurred. He reported low pitched tinnitus, which was described as very disturbing, in opposition to the high pitched but of very mild intensity remaining tinnitus which was a residual symptom of the first episode. PTA showed a decrease of the low frequencies similar to the one of the previous episodes (Figure 1B). He received $24 \mathrm{mg}$ of methylprednisolone p.o. tapered over eleven days. Low frequencies were restored to normal except for a mild loss remaining at $250 \mathrm{~Hz}$. High frequency thresholds remained unchanged except for a slight improvement at $4 \mathrm{KHz}$. Tympanometry was normal but in the left ear the stapedial reflex was partially produced. A brain MRI was ordered. The results showed VBD.

Auditory brainstem Responses (ABR) showed normal waveforms in both ears (Figure 6). Speech discrimination scores were normal. No vertigo was reported and the clinical vestibular examination and Videonystagmography was normal.

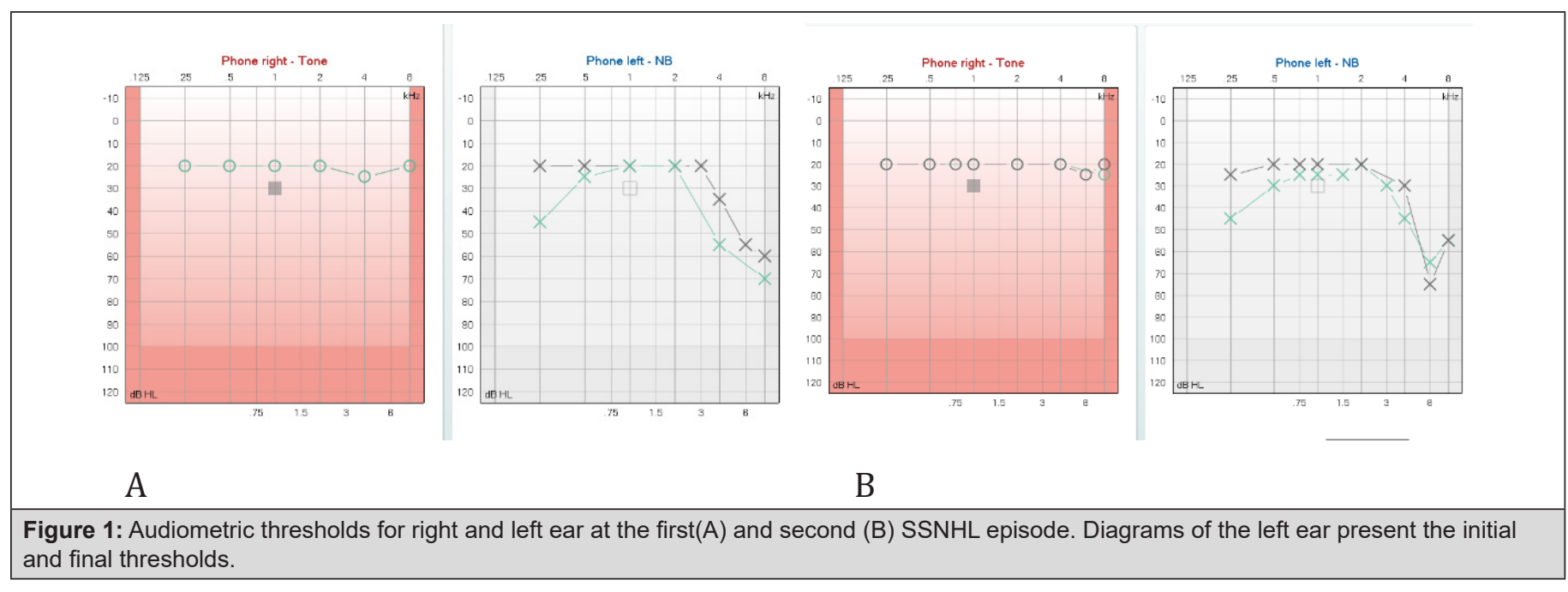



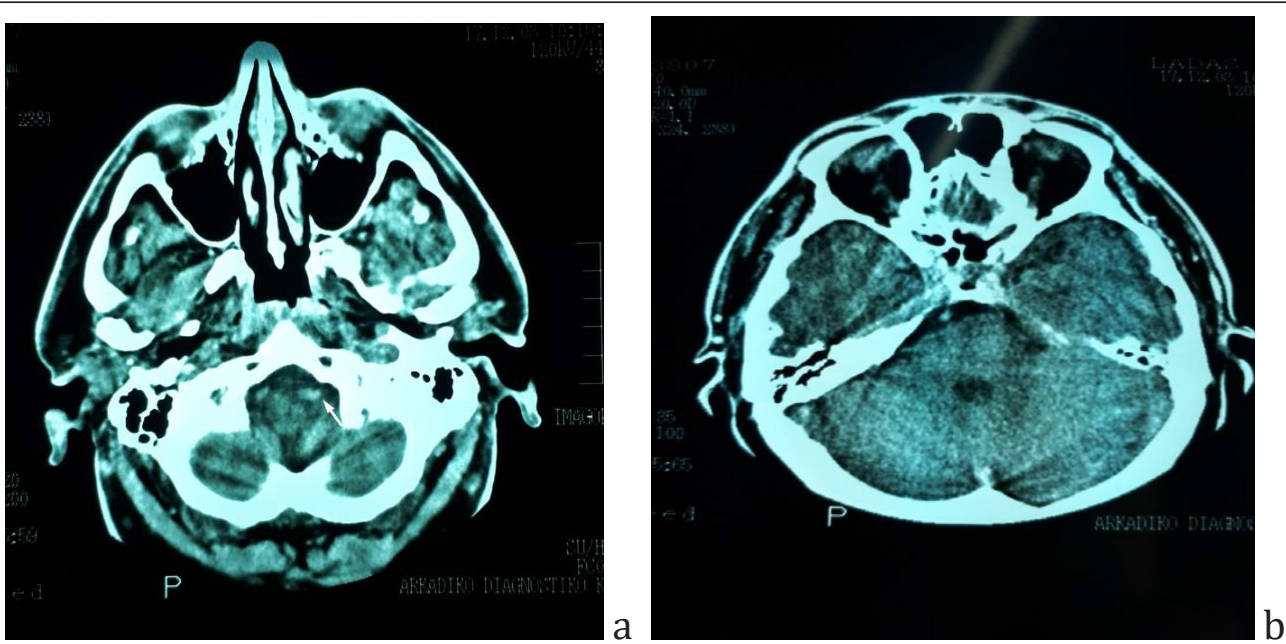

Figure 2: Brain CT scan of the patient sixteen years before the first episode of SSNHL, when he had a car accident. a) The enlargement and beginning tortuosity of the left vertebral artery is evident (arrow). b) The basilar artery has already a lateralized position at the right border of the dorsum sellae but it does not appear enlarged.
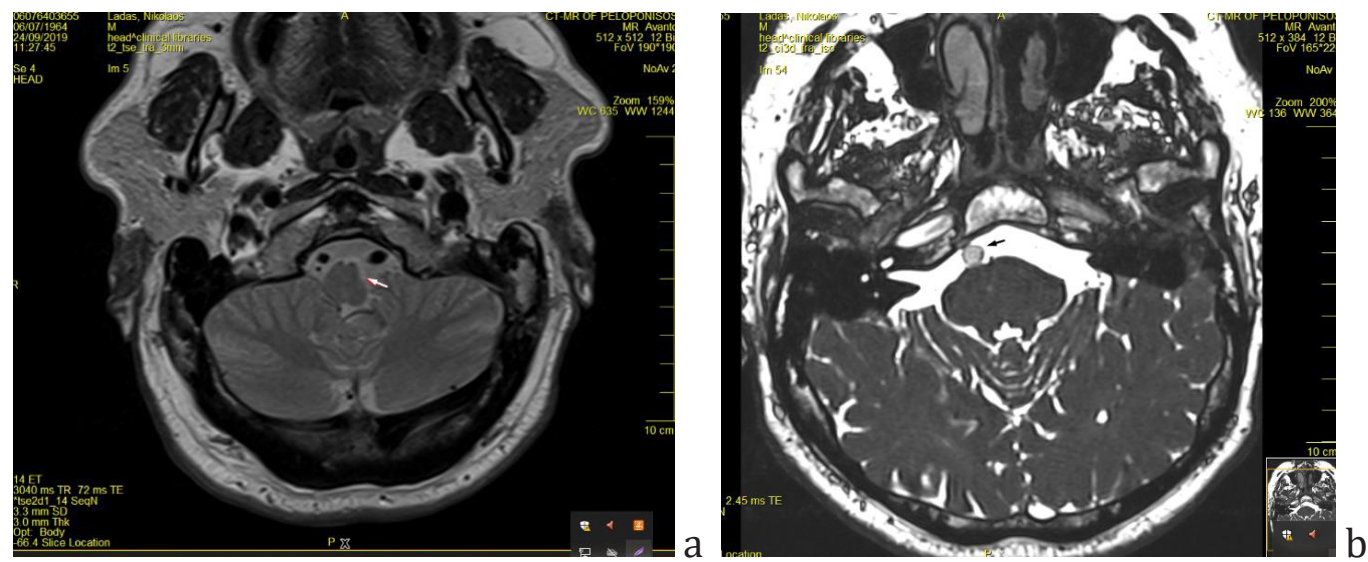

Figure 3: a) Axial T2-tse sequence showing the mass effect on the medulla oblongata (arrow) and the asymmetry of vertebral arteries, with left dominance. b) Axial section of Brain MRI (T2ci3d sequence) showing the basilar artery at the midpons level, assuming a lateral position at the margin of the clivus (arrow). The diameter of the artery is $5.12 \mathrm{~mm}$ ). Note the similarities with fig 2, imaging done sixteen years earlier.
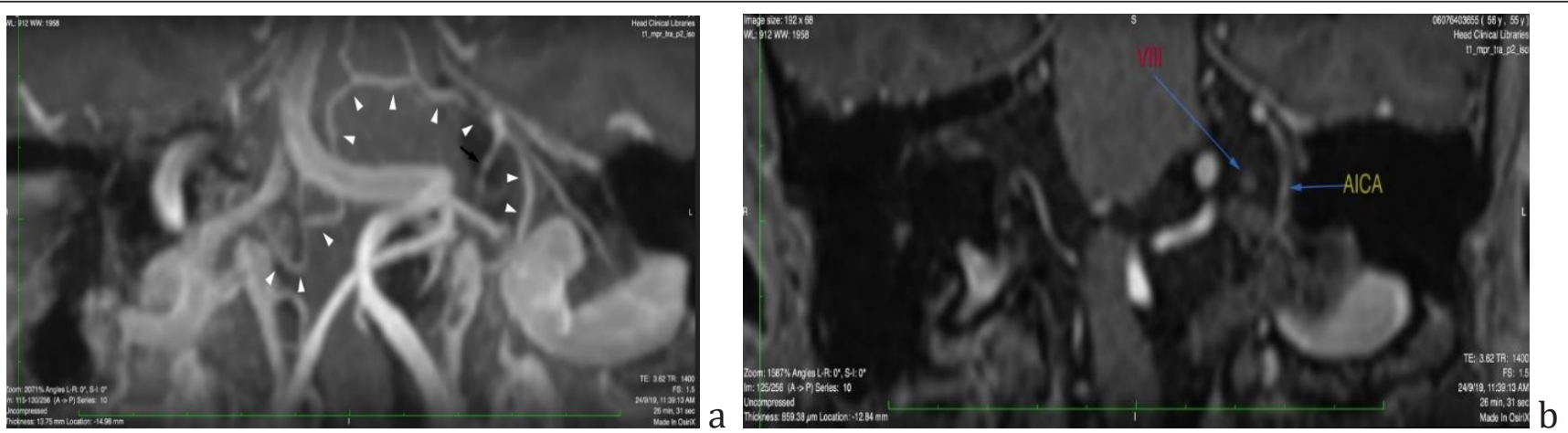

Figure 4: a) Oblique coronal view maximum intensity projection (MIP) reformatted T1-mpr-p2-iso MRI sequence showing the right vertebral artery crossing beneath the left dominant vertebral artery to merge in the left cerebellopontine angle and form the basilar artery, the first part of which is practically horizontal and then turns abruptly upwards at the right lateral border of the clivus. Right and left AICAs are marked with white arrowheads. The left AICA emerges with a paradoxically vertical proximal end, curves smoothly and then splits into the labyrinthine (black arrow), medial and lateral cerebellar branch. Anastomosing branches with other pontine arteries are evident b) Note the anatomical relationship of the VIIIth nerve with the labyrinthine branch of the AICA pictured in a. 


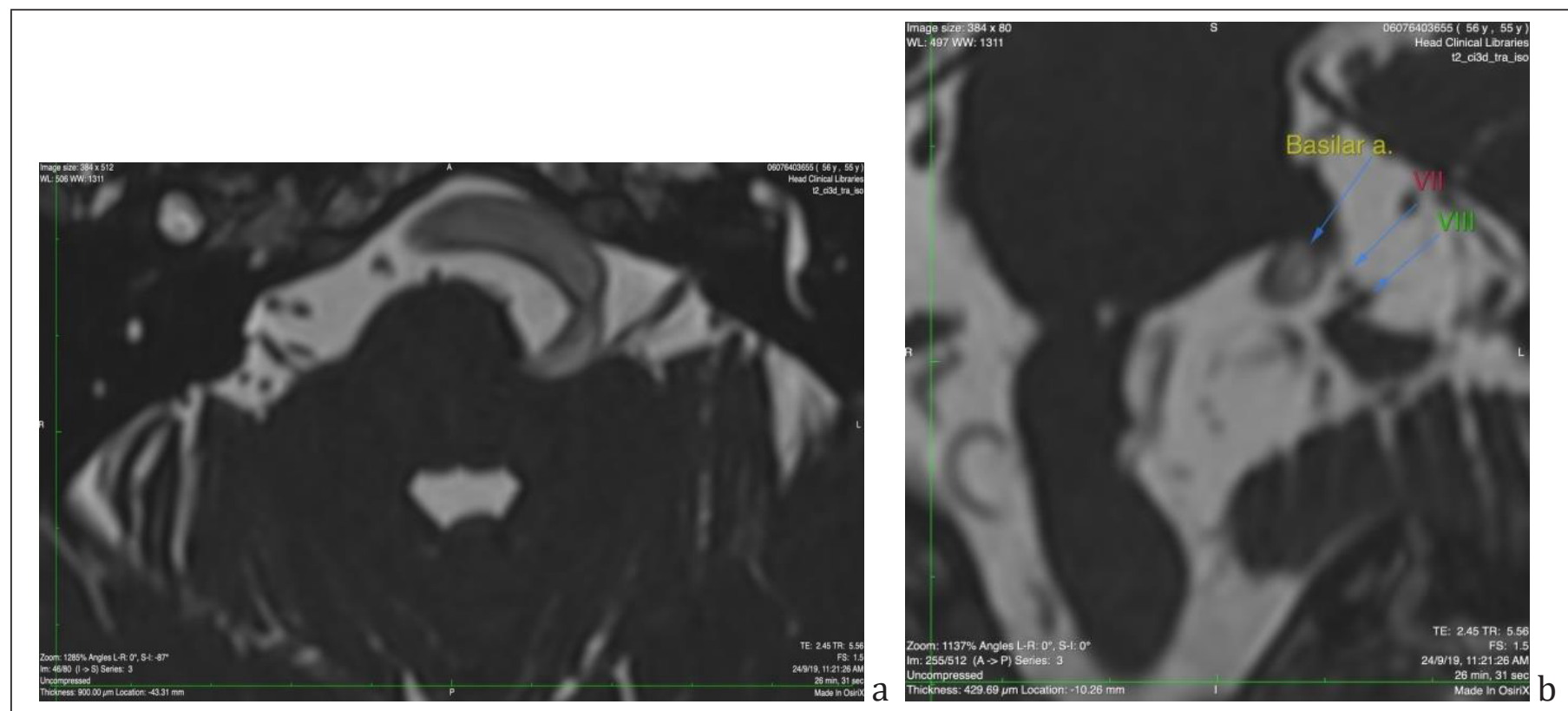

Figure 5: a) Axial section of Brain MRI (T2 ci3d sequence) showing the proximity of vertebrobasilar artery tortuous pathway with the root of the eighth cranial nerve. b) Coronal reconstruction of the same image, picturing in detail the relationship among the BA the VII and VIIIth cranial nerves. The distance between BA and VIIIth nerve was measured $1.8 \mathrm{~mm}$.

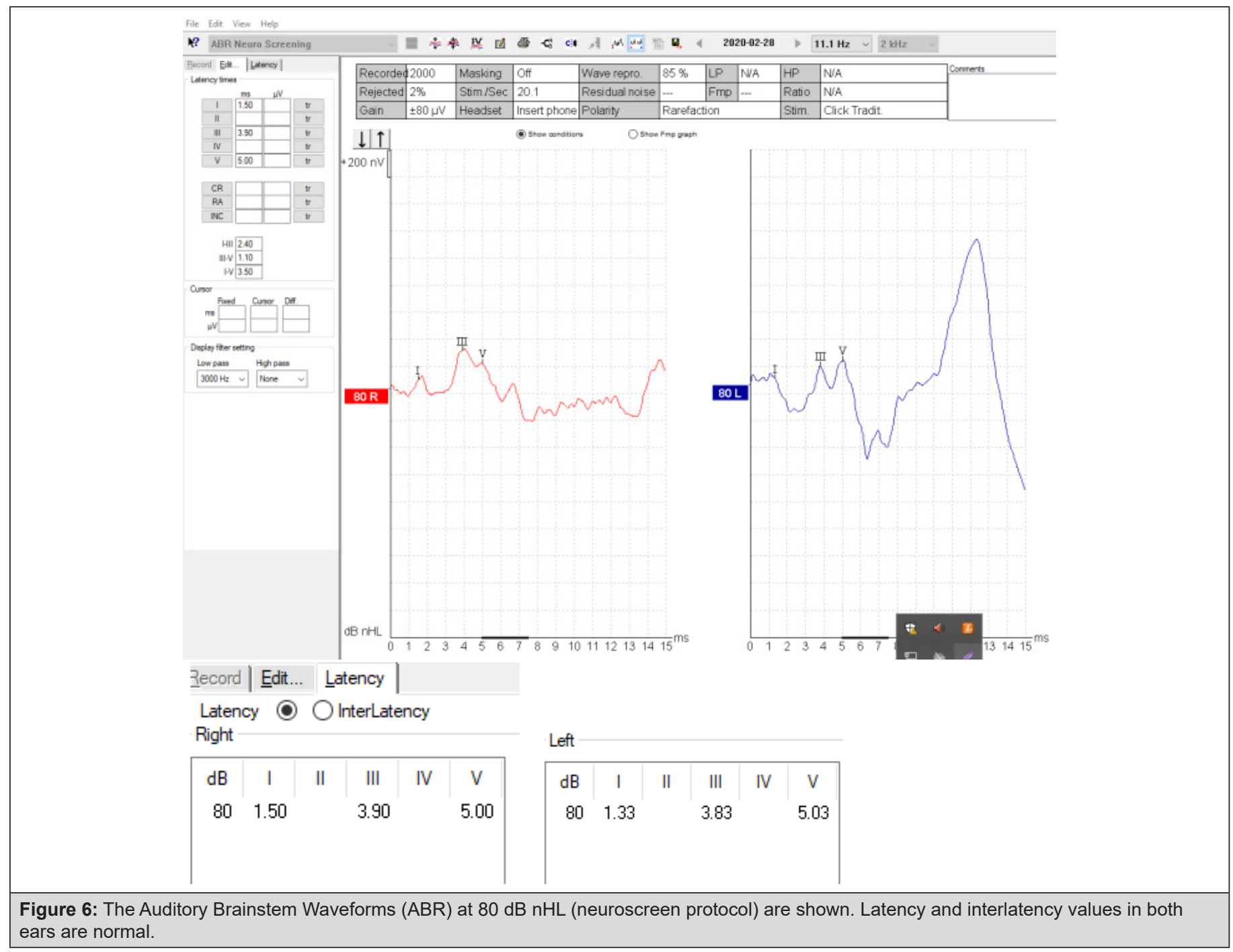


Sixteen years before the first episode, the patient had had a car accident for which he had a brain CT scan which was otherwise normal apart from an incidental comment about the existence of a tortuous left vertebral artery (Figure 2). MRI imaging findings of the vertebrobasilar segment are shown on Figures $3 \& 4$.

According to Smoker's criteria BA reaches the lateral border of the clivus (Laterality criterion grade 2) (Figure $3 \mathrm{~b}$ ). The level of bifurcation reaches the floor of the third ventricle (grade 2). The dimension of the BA at the midpons level is $5.12 \mathrm{~mm}$. (Smoker's criteria for ectasia $>4.5 \mathrm{~mm}$ ). In the present case the vertebrobasilar system is tortuous and elongated with moderate ectasia. No signs of intraluminal thrombi are found. There is a mass effect on the brainstem and a dominance of the left vertebral artery (side to side difference of vertebral artery diameters was $>0.3 \mathrm{~mm}$ ) [7] (Figure 3a).

Figure 5 shows the proximity of the vertebrobasilar artery to the eighth cranial nerve. The question arising is whether SSNHL episodes could be attributed to compression of the nerve root by the artery. The distance between the artery and the nerve has been measured to be $1.8 \mathrm{~mm}$.

The patient was referred to neurosurgical clinic, where he was given the instruction of careful vigilance about his blood pressure and cholesterol. Five months later the patient had a follow-up audiogram which did not show any change compared to the last audiogram of the second incident. Low pitched tinnitus ceased and he reported only the usual mild high pitched tinnitus on the left.

\section{Discussion}

Sudden Sensorineural Hearing Loss (SSNHL) has been defined as the hearing loss of at least $30 \mathrm{~dB}$ in three consecutive frequency bands occurring over no more than 72 hours [8]. Annual incidence rate ranges from 4 cases over 100.000 population to as many as 160 cases per 100.000 population [9]. Vascular events such as vasospasm, thromboembolism, and weak blood circulation have been named among the pathomechanisms responsible for this syndrome [10]. The inner ear receives blood supply by the labyrinthine artery (or arteries as in $2 / 3$ of individuals there are two labyrinthine arteries perfusing the inner ear) deriving from the Anterior Inferior Cerebellar Artery (AICA) and in fewer cases from the Basilar Artery [11].

Recurrence of SSNHL is rare [12], unless there are underlying systemic, autoimmune or neurologic disorders [13]. Fushiki et al. [14] claim that recurrent SSNHL involving the low frequencies could be an early sign of Menière's disease in case of concurrent spontaneous nystagmus and ECochG findings. The present case is a little more intriguing as in the first incident of hearing loss both the low and high frequencies were involved with a partial improvement of the high frequencies and full recovery of the low ones. The second incident simulates more a cochlear hydropic event as it affected only the low frequencies.

In the presence of Vertebrobasilar Dolichoectasia the reasonable question arising was whether Sudden Sensorineural Hearing Loss could be attributed to Posterior circulation issues given the vascular morphological alterations which are not negligible. The first pathophysiological mechanism would be a mass effect compressing or stretching the eighth nerve either by direct contact or regional oedema. Following thorough investigation of MRI sections (Figure $4 \& 5$ ) this possibility is rather eliminated. No direct compression of the nerve is seen. Supporting this are the normal auditory brainstem responses (although from experience with vestibular schwannoma, minor compressive effects can be missed by this diagnostic modality). Audiology results did not reveal retrocochlear hearing loss, apart from a partially released stapedial reflex which was not investigated any further. However, in a study by Sauvaget et al. [15] focusing on vertebrobasilar insufficiency and SSNHL, they claim that the lack of retrocochlear features of hearing loss, does not preclude a central cause in the SSNHL. Even in studies where compression of the nerve is more evident, authors claim that the correspondence between compression site and symptoms is not always obvious [16,17].

Things get even more complicated as in studies with large samples of patients with SSNHL and posterior circulation anatomical variations (VBD, vascular loop) Maruyama et al. [6] found no correlation between the laterality of the above configurations of the arteries and the affected side, whereas Kim et al. [18] found the laterality of BA curvature and laterality of SSNHL to be inversely associated in a population sample in which right sided BA curvature and left sided hearing loss prevailed. They used modified Smoker criteria which was not the case in the study of Maruyama et al. [6] who simply mentioned the existence of BA curvature without grading it. Our patient fulfilled the Smoker criteria of a markedly tortuous BA (Smoker laterality 2) and coincided with Kim's findings of a right sided BA curvature and left sided hearing loss. Additionally, he had a severely tortuous VA causing the mass effects on the brainstem (Figure 3a). According to Kim et al. [18] SSNHL can be due to blood circulation disturbances in a stretched AICA deriving from a basilar artery which is curving contralaterally. Sunwoo [19] sustains the same hypothesis as in his study the severity of BA tortuosity correlated with occurrence of severe sudden hearing loss on the opposite side. Moreover, the pathomechanism of low wall sheer stress in the inner wall of a curved basilar artery and traction of the pontine perforators leading to formation of microthrombi is a possible explanation of the fact that pontine infarctions are more frequent on the side contralateral to BA curvature [20].

In the present case the left AICA emanates in a direction perpendicular to the typical horizontal one, which could be a factor leading to blood flow disturbances. However as seen in Figure 4a 
the presence of anastomoses with neighboring pontine vessels, as described by the cadaveric study of Nishijima [21] could be a possible reason that a major ischemic event has not taken place. To what extent such a vascular configuration could be responsible for a moderate cochlear ischemia leading to a moderate hearing loss in a limited area of the cochlea, is highly speculative.

One of the unique features of the present study is the existence of an imaging modality sixteen years before the occurrence of SSNHL. Although it is not an MRI but a CT, the finding of a tortuous left vertebral artery had been pinpointed even at that time of the past. The basilar artery appeared lateralized at the level of upper pons but no ectasia was apparent yet at that time. In none of the relevant studies has there been a previous imaging report to testify the longevity and/or the pathogenesis of VBD. The asymmetric vertebral artery flow contributes to the development of basilar artery curvature in the side opposite to the side of VA dominancy. There are references about the existence of a dominant vertebral artery more frequently on the left side [18-20]. Despite the fact that hyperlipidemia and hypertension were present in the patient's recent medical history, (of a few years) the lateralization of the BA being evident many years earlier (Figure $2 b$ ) is in accordance with the pathogenetic theory of a mechanism other than atherogenesis, involving a disfunction of matrix metalloproteinases leading to aberrant vascular remodeling and abnormal connective tissue in the arterial wall $[3,22]$.

The second unique feature of the case presented is the configuration of the audiogram in the initial incident affecting the lower and higher frequencies and preserving the mid ones followed by a partial recovery, whereas recurrence affected mainly the low frequencies. Recurrence ofSSNHL is unusual and typical audiometric patterns are not available in relevant studies [12,23,24]. Cochlear hydrops cannot be precluded but high frequency involvement on the initial attack is atypical. Huh et al. [17] describe fluctuating hearing loss in one of VBD patients of their series, but they do not become more specific about the features of fluctuation. The finding of a markedly tortuous vertebrobasilar artery is a factor that has to be taken into account, but as direct nerve compression is not obvious on MRI, a further investigation with autoimmune and clotting profile of the patient should be done.

\section{Conclusion}

This is a case report adding to the few case series that already exist in the bibliography, investigating the relationship between Vertebrobasilar Dolichoectasia and Sudden Sensorineural Hearing Loss. Tortuosity of the vessels is important affecting both the left vertebral and the basilar artery, a finding coinciding with the majority of previous references. Although there is no evidence of immediate compression of the eighth nerve from the vertebrobasilar artery, the possibility of high frequency hearing loss failure to recover, being a result of VBD on the nerve and cochlea blood supply, cannot be excluded. Low frequency hearing loss and threshold restoration to normal is even more intriguing. Nevertheless, fluctuating hearing loss has already been reported in VBD series and of course the possibility of cochlear hydrops is always existent. Vertebrobasilar Dolichoectasia is by itself a potentially harmful clinical entity and awareness of its existence is by all means beneficiary to the patient.

\section{Conflict of Interest}

The authors declare no conflicts of interest.

\section{References}

1. Smoker WR, Price MJ, Keyes WD, Corbett JJ, Gentry LR. (1986) High-resolution computed tomography of the basilar artery: 1. Normal size and position. AJNR Am J Neuroradiol 7(1): 55-60.

2. Smoker WRK, Corbett JJ, Gentry LR, Keyes WD, Price MJ, et al. (1986) High-resolution computed tomography of the basilar artery: 2. Vertebrobasilar dolichoectasia. Clinical-pathological correlation and review. AJNR Am J Neuroradiol 7: 61-72.

3. Samim M, Goldstein A, Schindler J, Johnson M (2016) Multimodality Imaging of Vertebrobasilar Dolichoectasia: Clinical Presentations and Imaging Spectrum. Radiographics 36(4): 1129-1146.

4. Wolters FJ, Rinkel GJE, Vergouwen MDI (2013) Clinical course and treatment of vertebrobasilar dolichoectasia: A systematic review of the literature. Neurol Res 35(2): 131-137.

5. Passero S, Rossi S (2008) Natural history of vertebrobasilar dolichoectasia. Neurology 70(1): 66-72.

6. Maruyama A, Kawashima Y, Fujikawa T, Makabe A, et al. (2020) Potential Confounding Factors May Influence the Association Between Configurations of the Vertebrobasilar System and the Incidence of Idiopathic Sudden Sensorineural Hearing Loss and Canal Paresis. Otol Neurotol 41(5): 548-555.

7. Li Q, Xie P, Yang W S, Yan B, Davis S, et al. (2019) Vertebral Artery Compression Syndrome. Front Neurol 10: 1075.

8. Rauch SD (2008) Clinical practice. Idiopathic sudden sensoneurinal hearing loss. N Eng J Med 359(8): 833-840.

9. Hsu YH, Hu HY, Chiu YC, Lee FP, Huang HM (2016) Association of Sudden Sensorineural Hearing Loss with Vertebrobasilar Insufficiency. JAMA Otolaryngology-Head \& Neck Surgery 142 (7): 672-675.

10. Isabelle Mosnier 1, Alain Stepanian, Gabriel Baron, Camille Bodenez, Alain Robier, et al. (2011) Cardiovascular and thromboembolic risk factors in idiopathic sudden sensorineural hearing loss: a case-control study. Audiol Neurootol, 16(1): 55-66.

11. Hendrix P, Griessenauer C, Foreman P, et al. (2014) Arterial Supply of the Lower Cranial Nerves: A Comprehensive Review. Clinical Anatomy 27: 108-117.

12. Pecorari G, Riva G, Bruno G, Naqe N, Nardo M et al. (2020) Recurrences in Sudden Sensorineural Hearing Loss: A Long-Term Observational Study. Am J Audiol 29(1): 18-22.

13. G Conte, F Di Berardino , C Sina, D Zanetti, E Scola, et al. (2017) MR Imaging in Sudden Sensorineural Hearing Loss. Time to Talk. AJNR Am J Neuroradiol 38(8): 1475-1479.

14. Fushiki H, Junicho M, Aso S, Watanabe Y (2009) Recurrence rate of idiopathic sudden low-tone sensorineural hearing loss without vertigo: a long-term follow-up study. Otol Neurotol, 30 (3): 295-298.

15. E Sauvaget, S Kici, B Petelle, R Kania, H Chabriat, Ph Herman, et al. (2004) Vertebrobasilar occlusive disorders presenting as sudden sensorineural hearing loss. Laryngoscope, 114(2): 327-332. 
16. Daniels J, Rudic M (2020) Vertebrobasilar dolichoectasia: A rare presentation to the otolaryngologist. J Case Rep Images Otolaryngol 1:100001Z18JD2020.

17. Gene Huh, Yun Jung Bae, Hyun Jun Woo , Jung Hyun Park, Ja Won Koo, et al. (2020) Vestibulocochlear symptoms caused by vertebrobasilar dolichoectasia. Clin Exp Otorhinolaryngol 13(2): 123-132.

18. Chulho Kim, Jong Hee Sohn, Min Uk Jang, Sung Kwang Hong, Joong Seob Lee, et al. (2016) Ischemia as a potential etiologic factor in idiopathic unilateral sudden sensorineural hearing loss: Analysis of posterior circulation arteries. Hear Res 331: 144-151.

19. Sunwoo W (2020) Basilar artery tortuosity as a predictive factor for the efficacy of heparin adjuvant therapy in unilateral idiopathic sudden sensorineural hearing loss. Braz J Otorhinolaryngol S1808-8694(20): 30117-30118.

20. Hong JM, Chung CS, Bang OY, Yong SW, Joo IS, et al. (2009) Vertebral artery dominance contributes to basilar artery curvature and peri-vertebrobasilar junctional infarcts. J Neurol Neurosurg Psychiatry 80: 10871092
21. Nishijima Y (1994) Anatomical analysis of the basilar artery and its branches with special reference to the arterial anastomosis, and its course and distribution on the pontine ventral surface. Nihon Ika Daigaku Zasshi 61(6): 529-547.

22. Akgun V, Battal B, Bozkurt Y, Oz O, Hamcan S, et al. (2013) Normal Anatomical Features and Variations of the Vertebrobasilar Circulation and Its Branches: An Analysis with 64-Detector Row CT and 3T MR Angiographies. The Scientific World Journal 2013: 620162.

23. Pico F, Jacob MP, Labreuche J, Soufir N, Touboul PJ, Benessiano J et al (2010) Matrix metalloproteinase-3 and intracranial arterial dolichoectasia. Ann Neurol 67(4): 508-515.

24. Park IS, Kim YB, Choi SH, Hong SM (2013) Clinical analysis of recurrent sudden sensorineural hearing loss. ORL J Otorhinolaryngol Relat Spec 75(4): 245-249. 